\title{
APLICAÇÃO DO MOVIMENTO KEPLERIANO NA ORIENTAÇÃO DE IMAGENS HRC - CBERS 2B
}

\author{
Application of Kepler motion in the orientation of HRC-CBERS $2 B$ images \\ TIAGO LIMA RODRIGUES $^{1}$
LUIZ DANILO DAMASCENO FERREIRA $^{2}$ \\ ${ }^{1}$ Instituto Federal de Educação, Ciência e Tecnologia de Goiás - IFG \\ Campus Jataí - Rua Riachuelo, 2.090, Samuel Graham \\ CEP 75804-020 - Jataí - GO - Brasil \\ ${ }^{2}$ Universidade Federal do Paraná - UFPR \\ Curso de Pós-Graduação em Ciências Geodésicas - CPGCG \\ Caixa Postal 515 - Centro Politécnico - Jardim das Américas \\ CEP 12201-970 - Curitiba - PR - Brasil \\ engtlrodrigues@yahoo.com.br; luizdanilo@ufpr.br
}

\begin{abstract}
RESUMO
Nos últimos 20 anos, pesquisas voltadas ao desenvolvimento de modelos rigorosos para a orientação de sensores orbitais puhbroom lineares vêm sendo desenvolvidas e apresentadas. Na maioria destas pesquisas, a trajetória e a orientação do satélite durante a formação das cenas são obtidas a partir de polinômios de $1^{\circ}, 2^{\circ}$ e até $3^{\circ}$ grau. Porém, a atribuição de significado físico aos coeficientes polinomiais indica que o primeiro e o segundo termo se referem à velocidade e a aceleração da plataforma no instante referente à aquisição da primeira linha da cena. Estas quantidades podem ser associadas ao Problema dos Dois Corpos, sendo desenvolvido de acordo com a equação do Movimento Uniformemente Variado. O modelo resultante deste desenvolvimento foi denominado por Michalis e Dowman como Modelo de Kepler. Nesta pesquisa, o Modelo de Kepler é aplicado na orientação de imagens HRC/CBERS 2B e comparado com os modelos que utilizam polinômios para a propagação dos Parâmetros de orientação exterior (POE), amplamente utilizados atualmente. Os resultados obtidos ao comparar o Modelo de Kepler e os modelos polinomiais indicaram que o uso do primeiro modelo permitiu a obtenção de melhores resultados em relação ao segundo.

Palavras-chave: Orientação exterior; HRC/CBERS 2B; Modelo de Kepler.
\end{abstract}


Over the past 20 years, research aimed at developing of rigorous models for the orientation of linear pushbroom sensors aboard orbiting platforms have been developed and presented. In most studies, the trajectory and orientation of the satellite during the formation of different lines of a scene has been obtained from polynomials of $1^{\text {st }}, 2^{\text {nd }}$ and even $3^{\text {rd }}$ degree. However, the assignment of physical significance to the polynomial coefficients indicates that the first and the second terms refer to the velocity and acceleration of the platform at acquisition time of the first line of the scene respectively. In this case, these quantities can be associated whit the Two Bodies Problem which is developed according to the equation of Uniformly Varied Motion. The resulting model was named by Michalis and Dowman as Kepler Model. In this research, the Kepler Model is applied to the orientation of HRC/CBERS 2B images and compared to models using polynomials for the spread of exterior orientation parameters (POE), widely used today. The results obtained by comparing the Kepler model and polynomial models indicated that use of the first model allowed to obtain better results when compared to the second.

Keywords: Exterior Orientation; HRC/CBERS 2B; Kepler Model.

\section{INTRODUÇÃO}

Com a tendência cada vez maior de disponibilidade de dados de melhor resolução espacial, novas aplicações, como planejamento urbano, mapeamento em escalas médias, dentre outras, que antes eram atendidas apenas com imagens aéreas tornam-se possíveis utilizando-se imagens orbitais.

Neste contexto, mesmo estando fora de operação desde maio de 2010, as imagens obtidas pelo sensor HRC do satélite CBERS 2B se apresentam como uma alternativa para mapeamentos, em determinadas escalas, tendo em vista a resolução espacial fornecida aos usuários de $2,5 \mathrm{~m}$ e a disponibilidade gratuita para a comunidade de usuários.

No entanto, para que haja a possibilidade de extração de informações geodésicas e/ou cartográficas destas imagens, como por exemplo, coordenadas, feições e áreas, é necessário que haja a orientação exterior, isto é, a definição das posições e ângulos de atitude da plataforma durante a formação da cena, e a orientação interior das mesmas.

Os métodos de orientação indireta de imagens são aplicados a partir de uma modelagem matemática que relaciona elementos do espaço imagem com elementos do espaço objeto. Ao longo das últimas décadas, diversos modelos matemáticos de orientação de imagens têm sido desenvolvidos, sendo divididos conforme a complexidade, exatidão e aplicabilidade, em duas classes, a saber: modelos rigorosos, baseados no princípio da Colinearidade; e modelos aproximados, baseando-se em transformações polinomiais (TOUTIN, 2004). 
Os modelos rigorosos são indicados pela literatura como os que apresentam resultados potencialmente mais acurados (JACOBSEN, 2007). Tais modelos objetivam reproduzir o processo físico de formação da cena, levando em conta as informações de geometria interna e externa do sensor. Entretanto, estes modelos são utilizáveis apenas em imagens que não sofreram nenhum tipo de correção geométrica a priori e a imagens inteiras, ou seja, sem recorte.

Nos modelos denominados rigorosos, as Equações de Colinearidade recebem uma modificação em relação à modelagem efetuada em fotografias aéreas. Tal fato ocorre devido à natureza dinâmica da formação da cena, uma vez que as imagens do tipo pushbroom são formadas pela junção das várias linhas CCD do sensor, e, cada linha da cena é tomada de uma posição e orientação diferentes, ao contrário das imagens obtidas por câmaras de quadro (frame). Desta forma, há uma preocupação com a modelagem das mudanças da geometria de imageamento ocorridas durante o processo de geração da cena, ou seja, que melhor descreva as variações da posição e orientação da plataforma, variantes no tempo de formação da cena.

Nos últimos 25 anos, pesquisas desenvolvidas neste sentido propuseram diferentes maneiras de modelar as mudanças nos parâmetros de orientação exterior (POE) das plataformas orbitais durante a formação da cena, e relacioná-los ao princípio de Colinearidade. Como exemplos, citam-se Gugan e Dowman (1988), Westin (1990), Orun e Natarajan (1994), Bang e Cho (2001), Kim e Dowman (2006), Medeiros (2007), Medeiros e Tommaselli (2009), e Marcato et al (2011), que propõem a utilização de polinômios de $1^{\circ}, 2^{\circ}$ e até $3^{\circ}$ grau para modelagem das mudanças nos POE. Por outro lado há pesquisas baseadas na integração das Equações de Colinearidade com parâmetros de Mecânica Orbital. Dentre algumas destas pesquisas pode-se citar: Salamonowicz (1986), Kratky (1989), Radhadevi et al. (1998), Al-Rousan et al. (1997), Valadan e Petrie (1998), Fritsch e Stallman (2000), Chen e Teo (2002), Valadan e Sadeghian (2003), Baiocchi et al. (2004), Gianonne (2006).

Porém, ao realizar uma análise dos modelos que utilizam polinômios, Michalis e Dowman (2005) associaram os termos lineares com a velocidade da plataforma e os termos quadráticos com as acelerações. A partir desta consideração, foram desenvolvidos três modelos distintos utilizando diferentes métodos de propagação orbital, sendo que, em um dos modelos, denominado Modelo de Kepler, as acelerações podem ser estimadas a partir da solução do Problema dos Dois Corpos.

Diante do exposto, o objetivo deste artigo foi investigar a aplicação do Modelo de Kepler na orientação de imagens HRC - CBERS 2B e comparar os resultados mediante a aplicação de modelos que utilizam polinômios na modelagem da órbita. Cabe ressaltar, que mesmo este satélite estando fora de operação as metodologias aqui apresentadas são utilizáveis em outros sistemas de imageamento orbital, inclusive nas próximas missões CBERS. 


\section{TRABALHOS RELACIONADOS}

Dentro do contexto de orientação de imagens HRC - CBERS 2B, utilizando modelos rigorosos, alguns trabalhos podem ser citados. Tommaselli et al (2009) investigaram a fototriangulação de um par de imagens HRC, utilizando o modelo proposto por Orun e Natarajan (1994) e o modelo presente no aplicativo Leica Photogrammetry Suite (LPS). Medeiros et al (2009) aplicaram o modelo de coplanaridade usando feições retas para orientar uma imagem HRC, tendo como modelo de plataforma polinômios de $1^{\circ}$ grau. Oliveira et al (2011) realizaram uma fototriangulação em um par de imagens HRC, utilizando injunções relativas de posição da plataforma, com dados obtidos a partir dos arquivos .XML que acompanham as imagens. Os modelos de plataforma utilizados foram os de $1^{\circ}$ e $2^{\circ}$ grau, testando varias configurações de pontos de controle e de aplicação de injunções na posição e nos coeficientes polinomiais. Marcato et al (2011) avaliaram a orientação de uma imagem HRC utilizando dois modelos: o modelo de colinearidade com pontos considerando dados de órbita - MCPO (também denominado modelo órbita-atitude na literatura internacional) e o modelos de colinearidade com pontos - MCP, que é o modelo indicado por Orun e Natarajan (1994). Em Marcato (2011) e Marcato et al (2010) tem-se a fototriangulação em um bloco de 4 imagens HRC, utilizando os modelos MCPO e MCP. Nos experimentos, foram analisadas diversas configurações de pontos de controle e retas de controle.

Em todos os experimentos dos trabalhos citados, nota-se que a acurácia planimétrica investigada sobre os pontos de verificação é quase sempre maior que o valor de um pixel $(2,5 \mathrm{~m})$. Outro ponto ainda a ser destacado é que a componente $\mathrm{N}$ apresenta sempre a menor acurácia. Tais fatos estão associados basicamente a duas questões. A primeira relacionada a duas limitações radiométricas do sensor HRC (MEDEIROS et al, 2009; MARCATO et al, 2010), e a segunda relacionada à montagem das matrizes de detectores $\mathrm{CCD}$ no pano focal do sensor HRC (MARCATO et al, 2010; MARCATO, 2011).

Quanto à primeira questão, uma das limitações radiométricas do sensor HRC é o pequeno intervalo dinâmico de níveis de cinza das imagens, o que proporciona dificuldades no processo de identificação e medição de pontos nas mesmas, conforme indicado por Marcato (2011) a partir da Figura 1. Outra limitação radiométrica, que também influi na identificação e medição dos pontos nas imagens, segundo Carvalho et al (2009), é que o campo de visada instantâneo efetivo no terreno (EIFOV - Effective Instantaneous Field of View) do sensor HRC é de aproximadamente 4,1 e 4,6 m nas direções along e across-track respectivamente. $\mathrm{Na}$ teoria estes valores deveriam ser de aproximadamente $4,05 \mathrm{~m}$, uma vez que a resolução nominal é de $2,7 \mathrm{~m}$. Neste caso, a resolução efetiva na direção acrosstrack apresenta-se ligeiramente fora das especificações. Isto provoca uma pequena perda de resolução das imagens, produzindo o efeito de um pequeno borramento nas mesmas. 
Figura 1 - Imagem CBERS-2B HRC (a) Histograma da imagem; (b) Definição do ponto na imagem original (c) e na imagem com ampliação do contraste. Fonte: Marcato (2011).

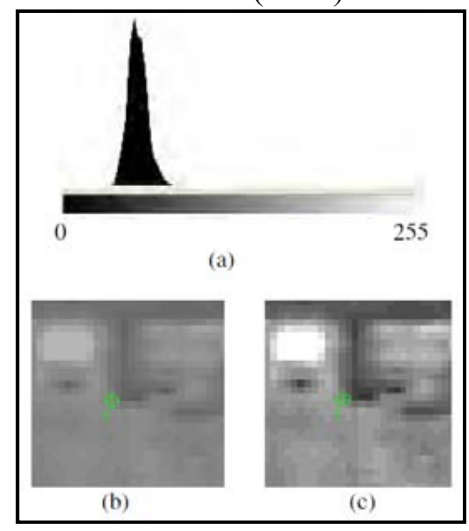

A segunda questão, relacionada à montagem das matrizes de detectores $\mathrm{CCD}$, e que está associada ao processo de construção da cena, é que há um desalinhamento entre as três matrizes que fusionadas formam a imagem final. As matrizes 1 e 3, mostradas na Figura 2, alinhadas entre si, distam no sentido de vôo de $26 \mathrm{~mm}$ da matriz 2, sendo que cada matriz possui 4096 elementos sensores.

Figura 2 - Geometria do plano focal do sensor HRC. Fonte: Epiphanio (2009)

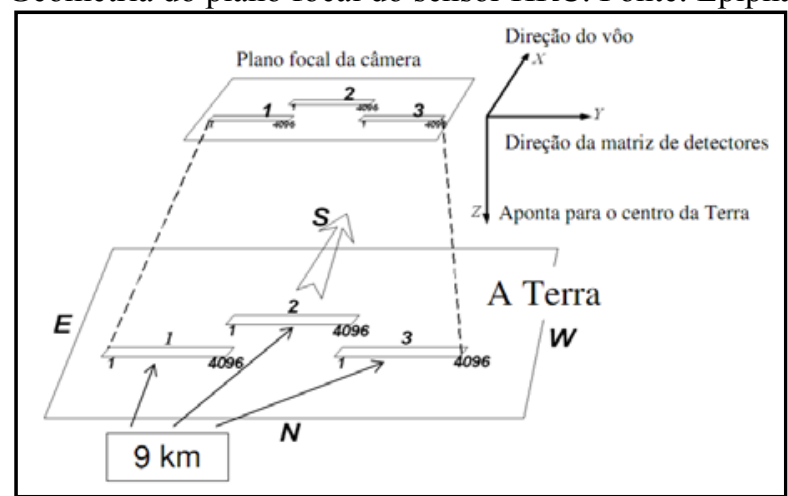

A junção da sub-cena central com as laterais é feita a partir de uma translação e de uma fusão, produzindo cenas de 12246 colunas disponibilizadas aos usuários em nível de processamento 1. Este procedimento, no entanto, gera um erro sistemático no modelo do sensor, uma vez que a matriz de detectores 2 é obtida em um instante diferente das demais matrizes na linha correspondente. 


\section{O MOVIMENTO KEPLERIANO}

As leis de Kepler descrevem a cinemática dos corpos celestes, porém, para entender como os corpos conseguem permanecer sempre na mesma trajetória, descrevendo as mesmas órbitas elípticas lança-se mão da lei da Gravitação Universal desenvolvida por Isaac Newton:

- Lei da Gravitação Universal de Newton (1687): “Duas partículas de massas $\mathrm{m}_{1} \mathrm{e}$ $\mathrm{m}_{2}$ atraem-se mutuamente segundo a linha que as une, com força diretamente proporcional ao produto de suas massas e inversamente proporcional ao quadrado da distância que as separa".

A forma vetorial da força é dada por:

$$
\vec{F}=-\frac{G m_{1} m_{2}}{r^{3}} \vec{r}
$$

onde $G$ é a constante gravitacional universal $\left(6,672 \times 10^{-11} \mathrm{~m}^{3} / \mathrm{Kg} . \mathrm{s}^{2}\right)$ e $r$ é a distância entre as partículas $\mathrm{m}_{1} \mathrm{e} \mathrm{m}_{2}$.

Restringindo o movimento a partir de duas massas $m_{1}$ e $m_{2}$ referenciadas em um sistema de coordenadas $\mathrm{X}_{\mathrm{i}}, \mathrm{Y}_{\mathrm{i}}, \mathrm{Z}_{\mathrm{i}}$, tem-se a situação descrita na Figura 3 a seguir:

Figura 3 - Esquema de forças em $\mathrm{m}_{1}$ e $\mathrm{m}_{2}$. Fonte: Adaptado de Seeber (2003).

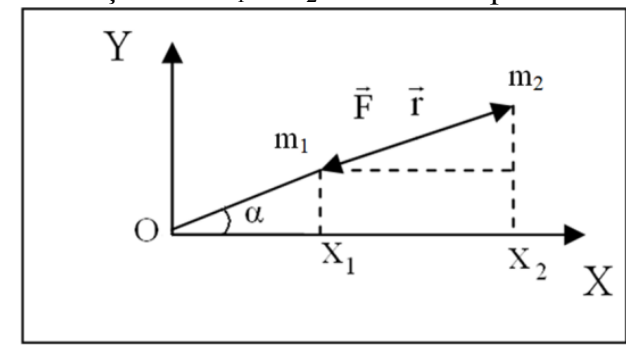

A partir da $2^{\mathrm{a}}$ Lei de Newton, adotando-se o movimento de $\mathrm{m}_{1}$ em relação à $\mathrm{m}_{2}$ e considerando como exemplo o plano OXY de forma a efetuar a dedução apenas para a componente $\mathrm{X}$ (Figura 3 ) tem-se que:

$$
F_{X_{1}}=m_{1} \ddot{X}_{1}
$$

onde $\ddot{X}_{1}$ é a aceleração da partícula de massa $\mathrm{m}_{1}$.

Reescrevendo a Equação 2 em função do ângulo $\alpha$ e do eixo X tem-se:

$$
F_{X_{1}}=-F \cos \alpha
$$

e do módulo da Equação 1, a Equação 3 torna-se: 


$$
m_{1} \ddot{X}_{1}=-F \cos \alpha=\frac{G m_{1} m_{2}}{r^{3}}\left(X_{2}-X_{1}\right) \text {. }
$$

Estendendo a Equação 4 para todas as componentes, adotando-se o movimento de $\mathrm{m}_{2}$ em relação à $\mathrm{m}_{1}$, dividindo-se posteriormente a equação resultante por $\mathrm{m}_{2}$, e vice-versa e, fazendo ainda o sistema de coordenadas coincidir com o centro de massa de $\mathrm{m}_{1}$, tem-se que (SEEBER, 2003):

$$
\begin{aligned}
& \ddot{X}=-\frac{G\left(m_{1}+m_{2}\right) X}{r^{3}}, \\
& \ddot{Y}=-\frac{G\left(m_{1}+m_{2}\right) Y}{r^{3}}, \\
& \ddot{Z}=-\frac{G\left(m_{1}+m_{2}\right) Z}{r^{3}} .
\end{aligned}
$$

No caso de um satélite terrestre de massa $\mathrm{m}_{2}$, esta pode ser negligenciada quando comparada com a massa da Terra $\mathrm{M}$ (e fazendo $\mathrm{m}_{1}=\mathrm{M}$ ), desta forma a Equação 5 escrita na forma vetorial fica:

$$
\overrightarrow{\ddot{r}}=-\frac{G M}{r^{3}} \vec{r}
$$

onde $\overrightarrow{\ddot{r}}$ é o vetor aceleração, $\vec{r}$ é o vetor posição da plataforma, $M$ é a massa da Terra e $\mathrm{G}$ é a constante gravitacional universal, de forma que por exemplo para o Elipsóide de Referência GRS80, $G M=3,986005 \times 10^{14} \mathrm{~m}^{3} \mathrm{~s}^{-2}$.

A Equação 6 é conhecida como Equação de Movimento e representa o movimento sujeito apenas ao campo gravitacional da Terra esférica, também chamado de Problema dos Dois Corpos, dado pelo Movimento Kepleriano. Este movimento é considerado uma primeira aproximação da órbita verdadeira do satélite, uma vez que não considera outras acelerações conhecidas como perturbações orbitais (SEEBER, 2003).

\section{MODELAGEM RIGOROSA PARA ORIENTAÇÃO DE IMAGENS OBTIDAS POR SENSORES PUSHBROOM}

Em sensores do tipo pushbroom linear, cada linha da cena é tomada em um momento diferente, ou seja, não há uma geometria do tipo perspectiva central única. Segundo Gupta e Hartley (1997), em intervalos regulares de tempo são obtidas imagens unidimensionais do plano de visada, e o conjunto destas constituem então uma imagem bidimensional.

Deste modo, a cena é formada de acordo com o movimento de varredura do sensor. No caso de plataformas orbitais, à medida que a plataforma se desloca ao longo da órbita, ocorre a formação da cena, que é a integração das diferentes linhas obtidas, formadas em diferentes posições do centro perspectivo e, consequentemente, com diferentes POE. 
Os modelos rigorosos geralmente são baseados no Princípio de Colinearidade, que indicam que os pontos no espaço imagem, espaço objeto e o centro perspectivo da imagem são colineares (Figura 4). Este princípio é modelado através das Equações de Colinearidade, que, no caso de sensores pushbroom lineares recebem uma modificação, na qual se inclui a modelagem das mudanças nos POE em função do tempo (GUGAN; DOWMAN 1988).

Figura 4 - Princípio de colinearidade aplicada a sensores lineares pushbroom.

Fonte: Adaptado de Galo (2006).

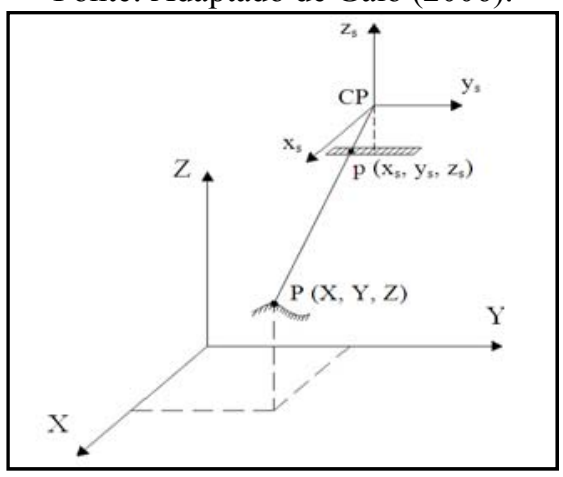

Com o objetivo de representar a geometria do sensor pushbroom linear, a componente $\mathrm{x}_{\mathrm{s}}$ é assumida como zero, visto que cada linha corresponde a uma imagem unidimensional gerada num dado instante de tempo, e o efeito de varredura da cena é devido ao movimento do satélite que se desloca ao longo desse eixo (GUGAN; DOWMAN, 1988). A Equação de Colinearidade modificada para sensores lineares pushbroom é apresentada a seguir:

$$
\begin{gathered}
0=x_{\mathrm{s}}=-f \frac{r_{11}\left(X_{i}-X_{S}\right)+r_{12}\left(Y_{i}-Y_{S}\right)+r_{13}\left(Z_{i}-Z_{S}\right)}{r_{31}\left(X_{i}-X_{S}\right)+r_{32}\left(Y_{i}-Y_{S}\right)+r_{33}\left(Z_{i}-Z_{S}\right)}, \\
y_{\mathrm{s}}=-f \frac{r_{21}\left(X_{i}-X_{S}\right)+r_{22}\left(Y_{i}-Y_{S}\right)+r_{23}\left(Z_{i}-Z_{S}\right)}{r_{31}\left(X_{i}-X_{S}\right)+r_{32}\left(Y_{i}-Y_{S}\right)+r_{33}\left(Z_{i}-Z_{S}\right)},
\end{gathered}
$$

sendo $X_{i}, Y_{i}$ e $Z_{i}$ coordenadas de um ponto $i$ qualquer no espaço objeto; $X_{S}, Y_{S}$ e $Z_{S}$ as coordenadas do centro perspectivo do sensor no Sistema de coordenadas cartesianas terrestre, as quais variam em função do tempo; $x_{s}, y_{s}$ são as coordenadas do Sistema de referência do sensor; $f$ é a distância focal do sensor e; $r_{11}, \ldots, r_{33}$ são os componentes da matriz de rotação em função dos ângulos de atitude da plataforma, $\omega, \varphi$ e $\kappa$, os quais também variam em função do tempo (Equação 9). 


$$
R=R_{Z}(\kappa) R_{Y}(\varphi) R_{X}(\omega)=\left[\begin{array}{lll}
r_{11} & r_{12} & r_{13} \\
r_{21} & r_{22} & r_{23} \\
r_{31} & r_{32} & r_{33}
\end{array}\right]
$$

Diversas abordagens distintas foram desenvolvidas para a modelagem das mudanças dos POE durante a formação da cena. O modelo apresentado por Gugan e Dowman (1988) apresenta o relacionamento entre os parâmetros de orientação exterior e o tempo $t$ é modelado a partir de polinômios de $2^{\circ}$ grau, apresentando um total de 18 parâmetros a serem estimados.

$$
\begin{gathered}
X_{s}=X_{0}+a_{1} t+b_{1} t^{2}, \\
Y_{s}=Y_{0}+a_{2} t+b_{2} t^{2}, \\
Z_{s}=Z_{0}+a_{3} t+b_{3} t^{2}, \\
\omega=\omega_{0}+a_{4} t+b_{4} t^{2}, \\
\varphi=\varphi_{0}+a_{5} t+b_{5} t^{2}, \\
\kappa=\kappa_{0}+a_{6} t+b_{6} t^{2},
\end{gathered}
$$

onde $X_{0}, Y_{0}$ e $Z_{0}$ são as coordenadas do centro perspectivo do sensor na primeira linha da imagem; $a_{1}, a_{2}, \ldots, b_{5}, b_{6}$ são os coeficientes polinomiais da modelagem de mudança nos POE e; $t$ é um dado instante de tempo da formação da cena.

Entretanto, segundo Orun e Natarajan (1994), uma vez que em um sensor pushbroom linear, certos parâmetros são altamente correlacionados entre si, a modelagem acima descrita pode apresentar soluções muito instáveis.

Mudanças em $\omega$ tem o mesmo efeito que pequenas mudanças em $Y_{s}$ e; pequenas mudanças em $\varphi$ tem o mesmo efeito de pequenas mudanças em $X_{s}$. Deste modo, determinações indiretas são instáveis, devido à alta correlação. Neste caso, há a necessidade de se considerar como constante ou $\omega$ ou $Y_{s}$ e $\varphi$ ou $X_{s}$.

As Equações 11 a seguir foram propostas por Orun e Natarajan (1994), onde os ângulos $\omega$ e $\varphi$ foram indicados como valores fixos e constantes, apenas com o ângulo $\kappa$ e as componentes da posição sendo propagados por polinômios de $2^{\circ}$ grau. A componente $x_{s}$, uma vez que é relacionada linearmente com o tempo, substitui este, de modo que e as mudanças em unidades (fator de escala entre $t$ e $x_{s}$ ) podem ser absorvidas pelos coeficientes polinomiais $a_{i}$ e $b_{i}$.

$$
\begin{gathered}
X_{s}=X_{0}+a_{1} x_{s}+b_{1} x_{s}{ }^{2}, \\
Y_{s}=Y_{0}+a_{2} x_{s}+b_{2} x_{s}{ }^{2}, \\
Z_{s}=Z_{0}+a_{3} x_{s}+b_{3} x_{s}{ }^{2}, \\
\kappa=\kappa_{0}+a_{4} x_{s}+b_{4} x_{s}{ }^{2} .
\end{gathered}
$$

Baseado no princípio que a propagação da posição da plataforma é modelada através de um polinômio de segundo grau, Michalis e Dowman (2005) e Dowman et al (2011) analisaram fisicamente o processo de formação da cena, e associaram os 
coeficientes lineares com a velocidade angular da plataforma e os termos quadráticos com as acelerações angulares.

A partir destas considerações, as acelerações utilizadas nos polinômios de segundo grau podem ser calculadas a partir da equação do Movimento Kepleriano e as posições da plataforma determinadas a partir da teoria do Movimento uniformemente variado (MUV). O modelo de plataforma é dado por:

$$
\begin{gathered}
X_{S}=X_{0}+u_{x} \tau-\frac{G M \cdot X_{0} \cdot \tau^{2}}{2 \cdot\left(X_{0}{ }^{2}+Y_{0}{ }^{2}+Z_{0}{ }^{2}\right)^{3 / 2}}, \\
Y_{S}=Y_{0}+u_{y} \tau-\frac{G M \cdot Y_{0} \cdot \tau^{2}}{2 \cdot\left(X_{0}{ }^{2}+Y_{0}{ }^{2}+Z_{0}{ }^{2}\right)^{3 / 2}}, \\
Z_{S}=Z_{0}+u_{z} \tau-\frac{G M \cdot Z_{0} \cdot \tau^{2}}{2 \cdot\left(X_{0}{ }^{2}+Y_{0}{ }^{2}+Z_{0}{ }^{2}\right)^{3 / 2}}, \\
\omega=\omega_{0}, \\
\varphi=\varphi_{0}, \\
\kappa=\kappa_{0}
\end{gathered}
$$

onde $u_{x}, u_{y}, u_{z}$ são as componentes do vetor velocidade; $\tau$ é o tempo associado à aquisição da primeira linha da cena.

Neste modelo é considerado que o tempo de aquisição de cada uma das linhas da cena é constante e a orientação das imagens deve ser realizada em um Sistema de coordenadas geocêntrico inercial. Originalmente, em Michalis e Dowman (2005), nota-se que os ângulos de atitude são considerados invariáveis durante o processo de formação das cenas. Porém, esta configuração pode ser alterada mediante o modo de aquisição de imagens de cada satélite. Um exemplo de alteração para cenas PRISMALOS pode ser visto em Dowman et al (2011).

\section{METODOLOGIA}

\subsection{Estudos de caso}

Com o objetivo de analisar os modelos propostos dois estudos de caso com configurações diferentes de pontos de controle foram realizados, em duas regiões.

A área de estudo 1 é localizada a leste do estado do Espírito Santo, englobando a capital Vitória e municípios adjacentes. A cena HRC/CBERS $2 \mathrm{~B}$ referente a esta área é a de órbita/ponto 148_B_123_1, de 24/02/2010. Esta imagem foi obtida a partir de um pedido especial, em virtude da necessidade da mesma apresentar nível de processamento 1 , ou seja, apenas com aplicação de correção radiométrica. A cena original foi recortada no sentido Norte - Sul.

Os pontos de controle (11) e de verificação (6) foram obtidos a partir das estações geodésicas do SGB presentes na área de estudo (17). A distribuição dos pontos de controle e verificação na cena recortada é apresentada na Figura 5 a seguir. Esta área de estudo apresenta quantidade reduzida de pontos de controle e verificação, os quais são distribuídos de forma não homogênea sobre a cena. Esta 
distribuição foi proposital, com o objetivo de se aproximar da realidade dos usuários que nem sempre dispõem de distribuição homogênea e em grande quantidade.

Figura 5 - Distribuição dos pontos de controle e verificação na cena recortada da área de estudo 1.

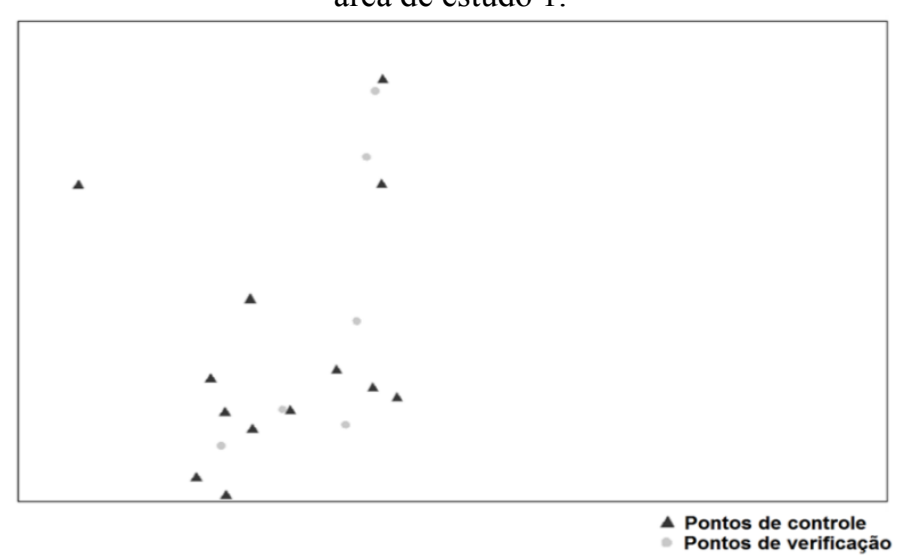

A área de estudo 2 é localizada na área central de Brasília - Distrito Federal. A cena HRC/CBERS 2B, em nível de processamento 1 utilizada foi a de órbita/ponto 157 B_118_1, de 05/06/2008, referente a esta área de estudo.

Os pontos de controle (70) e de verificação (43) nesta área foram obtidos a partir de cartas topográficas na escala 1:2000 do SICAD-DF em um total de 113 pontos (Figura 6).

Figura 6 - Distribuição dos pontos de controle e verificação na cena da área de estudo 2 .

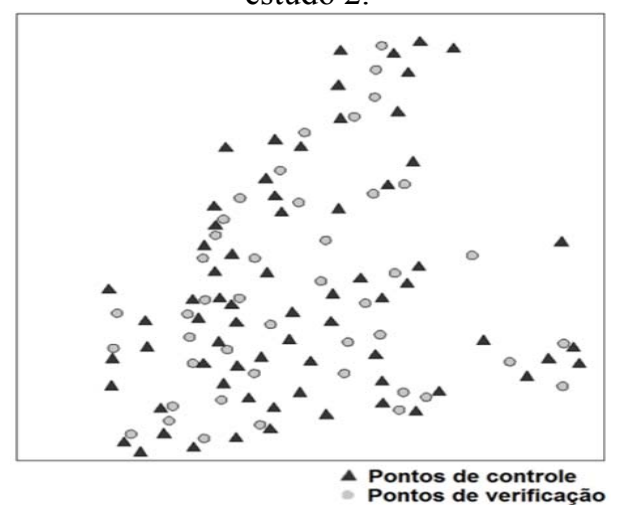

Bol. Ciênc. Geod., sec. Artigos, Curitiba, v. 19, nº 1, p.114-134, jan-mar, 2013. 
Em ambas as cenas a precisão adotada para as observações foi de 3 pixels, ou seja, $0,030 \mathrm{~mm}$, baseada em testes de repetição de medição, com o cálculo do desvio padrão. Foram analisadas 5 medições, utilizando o software ENVI 4.2, de quatro pontos em cada uma das duas imagens utilizadas, conforme é apresentado um exemplo na Figura 7 a seguir.

Figura 7 - Exemplo de repetição de medição de pontos de controle no espaço imagem para estimativa da incerteza associada.

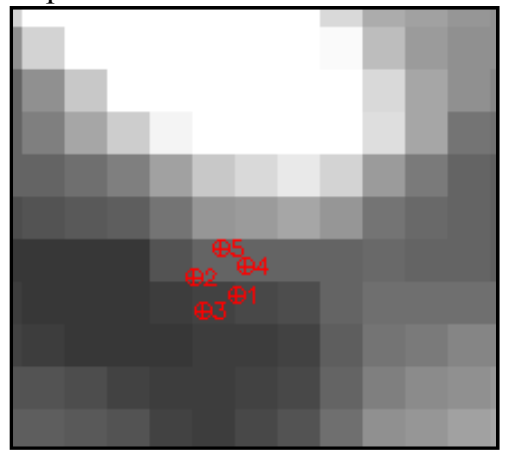

\subsection{Modelos para a orientação das imagens HRC - CBERS 2B}

Todos os modelos matemáticos de orientação nesta pesquisa partem das Equações de Colinearidade (Equações 7 e 8). A diferença entre os modelos analisados está nos modelos de plataforma utilizados. O primeiro modelo utiliza polinômios de $1^{\circ}$ grau:

$$
\begin{gathered}
X_{s}=X_{0}+a_{1} x_{s}, \\
Y_{s}=Y_{0}+a_{2} x_{s}, \\
Z_{s}=Z_{0}+a_{3} x_{s}, \\
\kappa=\kappa_{0}+a_{4} x_{s} .
\end{gathered}
$$

O segundo modelo de plataforma analisado é o indicado nas Equações 11 utilizando polinômios de $2^{\circ}$ grau, proposto por Orun e Natarajan (1994).

Nestes dois modelos matemáticos as coordenadas do espaço objeto foram utilizadas no Sistema de coordenadas UTM. Os ângulos $\omega$ e $\varphi$ receberam injunções absolutas por conta da correlação com $Y_{s}$ e $X_{s}$ respectivamente. Os valores destes ângulos para as cenas da área de estudo 1 e 2 foram obtidos a partir da média das informações contidas nos metadados das imagens (arquivos .xml), e constam na Tabela 1 a seguir. 
Tabela 1 - Ângulos $\omega$ e $\varphi$ obtidos a partir dos metadados para as cenas da área de estudo 1 e 2 .

\begin{tabular}{c|c|c|c|c}
\hline Cena & \multicolumn{2}{|c|}{ Área de estudo 1 } & \multicolumn{2}{c}{ Área de estudo 2 } \\
\hline \multirow{2}{*}{ Ângulos } & $\omega$ & $\varphi$ & $\omega$ & $\varphi$ \\
\cline { 2 - 5 } & $-0,625973^{\circ}$ & $-0,0135306^{\circ}$ & $-1,59543^{\circ}$ & $0,1088349^{\circ}$ \\
\hline
\end{tabular}

O terceiro modelo analisado nesta pesquisa é o Modelo de Kepler indicado nas Equações 12 e 13, proposto por Michalis e Dowman (2005). Neste modelo, as coordenadas do espaço objeto e das posições do satélite devem ser utilizadas no Sistema de coordenadas geocêntricas inerciais. Como citado anteriormente, no Modelo de Kepler apresentado em Michalis e Dowman (2005), os ângulos de atitude são incógnitos e considerados invariáveis durante o tempo de formação da cena. Porém, com a finalidade de adaptar o modelo de plataforma ao modo de aquisição de imagens do satélite CBERS 2B, considerando o denominado movimento de caranguejo do satélite (MARCATO, 2011), os ângulos $\omega$ e $\varphi$ foram mantidos invariáveis e os ângulos $\kappa$ foram propagados por polinômios de $1^{\circ}$ e $2^{\circ}$ grau. Deste modo, nesta pesquisa os Modelos de Kepler nas duas configurações apresentadas foram denominados de Modelo de Kepler 1 e 2.

Para a determinação dos parâmetros nos três modelos, utilizou-se o Método dos Mínimos Quadrados com o modelo paramétrico, tendo em vista que as equações dos modelos são organizadas de maneira a isolar as observações como função dos parâmetros de forma explícita. Os parâmetros estimados em cada modelo e os Parâmetros de orientação interior utilizados são indicados nas Tabelas 2 e 3 a seguir.

Tabela 2 - Parâmetros a serem estimados nos ajustamentos.

\begin{tabular}{c|c|c|c|c}
\hline \multirow{2}{*}{ Modelos } & $\begin{array}{c}\text { Modelo } \\
\text { Polinomial } \\
\text { de } \mathbf{1}^{\mathbf{0}} \text { grau }\end{array}$ & $\begin{array}{c}\text { Modelo } \\
\text { Polinomial de } \\
\mathbf{2}^{\mathbf{0}} \text { grau }\end{array}$ & $\begin{array}{c}\text { Modelo de } \\
\text { Kepler 1 }\end{array}$ & $\begin{array}{c}\text { Modelo de } \\
\text { Kepler 2 }\end{array}$ \\
\hline \multirow{3}{*}{ Parâmetros } & $X_{0}, a_{1}, Y_{0}, a_{2}$, & $X_{0}, a_{1}, b_{1}, Y_{0}$, & $X_{0}, Y_{0}, Z_{0}, u_{x}$, & $X_{0}, Y_{0}, Z_{0}, u_{x}$, \\
& $Z_{0}, a_{3}, \kappa_{0}$, & $a_{2}, b_{2}, Z_{0}, a_{3}$, & $u_{y}, u_{z}, \omega, \varphi, \kappa$, & $u_{y}, u_{z}, \omega, \varphi, \kappa$, \\
& $a_{4}$. & $b_{3}, \kappa_{0}, a_{4}, b_{4}$. & $a_{4}$. & $a_{4}, b_{4}$. \\
\hline
\end{tabular}

Tabela 3 - Parâmetros de orientação interior do sensor HRC. Fonte: Marcato (2011).

\begin{tabular}{l|c}
\multicolumn{1}{c|}{ Parâmetro } & Valor \\
\hline Distância focal (f) & $3398 \mathrm{~mm}$ \\
\hline Tamanho do pixel no CCD (PS) & $0,010 \mathrm{~mm}$ \\
\hline Número de colunas por linha CCD & 12246 \\
\hline Tempo de integração da linha CCD & $0,000345 \mathrm{~s} /$ linha \\
\hline
\end{tabular}




\section{RESULTADOS E DISCUSSÕES}

\subsection{Experimento 1}

O experimento 1 refere-se à apresentação dos resultados na área de estudo 1 . Este experimento caracteriza-se por uma quantidade reduzida de pontos de controle e verificação em uma cena com recorte na direção Norte - Sul. A Tabela 4 a seguir apresenta os resultados dos ajustamentos.

Tabela 4 - Resultados obtidos a partir dos ajustamentos na área de estudo 1.

\begin{tabular}{c|c|c|c}
\hline $\begin{array}{c}\text { Modelo } \\
\text { Polinomial de 1 } \\
\text { grau }\end{array}$ & $\begin{array}{c}\text { Modelo } \\
\text { Polinomial de 2 } \\
\text { grau }\end{array}$ & $\begin{array}{c}\text { Modelo de } \\
\text { Kepler 1 }\end{array}$ & $\begin{array}{c}\text { Modelo de } \\
\text { Kepler 2 }\end{array}$ \\
\hline$\hat{\sigma}_{0}^{2}=1,3953$ & $\hat{\sigma}_{0}^{2}=1,6479$ & $\hat{\sigma}_{0}^{2}=1,3674$ & $\hat{\sigma}_{0}^{2}=1,4626$ \\
$\chi^{2}$ calculado: & $\chi^{2}$ calculado: & $\chi^{2}$ calculado: & $\chi^{2}$ calculado: \\
19,534 & 16,479 & 16,408 & 16,089 \\
$\chi^{2}$ teórico & $\chi^{2}$ teórico & $\chi^{2}$ teórico & $\chi^{2}$ teórico \\
$(14 ; 0,975): 24,736$ & $(10 ; 0,975): 20,483$ & $(12 ; 0,975): 23,336$ & $(13 ; 0,975): 21,920$ \\
\hline RMSE $V x_{s}=0,033$ & RMSE $V x_{s}=0,029$ & RMSE $V x_{s}=0,030$ & RMSE $V x_{s}=0,030$ \\
mm & $m m$ & $m m$ & $m m$ \\
\hline RMSE $V y_{s}=0,025$ & RMSE $V y_{s}=0,024$ & RMSE $V y_{s}=0,023$ & $\mathrm{RMSE} V y_{s}=0,023$ \\
$\mathrm{~mm}$ & $\mathrm{~mm}$ & $\mathrm{~mm}$ & $\mathrm{~mm}$ \\
\hline
\end{tabular}

Para todos os modelos analisados o teste do qui-quadrado para a hipótese básica de $\hat{\sigma}_{0}^{2}=\sigma_{0}^{2}$ não é rejeitada ao nível de significância de 5\% (Tabela 4). Tal resultado indica que as ponderações nas observações foram adequadas e que os modelos matemáticos conseguiram representar a realizada física do processo. As acurácias dos resíduos obtidos apresentaram-se próximos ao valor da precisão adotada na coleta das observações $(0,030 \mathrm{~mm})$.

Posteriormente a avaliação da acurácia das orientações foi realizada, utilizando-se as Equações de Colinearidade inversas. Para o valor de $h_{i}$ foram utilizados os valores dos próprios pontos de verificação. A Tabela 5 a seguir apresenta os valores do RMSE para as componentes E e N do Sistema UTM para os três modelos.

Tabela 5 - Acurácia nas componentes $\mathrm{E}, \mathrm{N}$ dos pontos de verificação na área de estudo 1 para os modelos utilizados.

\begin{tabular}{c|c|c|c|c|c|c|c|c}
\hline \multirow{2}{*}{ Modelos } & \multicolumn{2}{|c|}{$\begin{array}{c}\text { Modelo de } \\
\text { Kepler 1 }\end{array}$} & \multicolumn{2}{c|}{$\begin{array}{c}\text { Modelo de } \\
\text { Kepler 2 }\end{array}$} & \multicolumn{2}{c|}{$\begin{array}{c}\text { Modelo } \\
\text { Polinomial de } \\
\mathbf{1}^{\mathbf{a}} \text { ordem }\end{array}$} & \multicolumn{2}{c}{$\begin{array}{c}\text { Modelo } \\
\text { Polinomial de 2 } \\
\text { ordem }\end{array}$} \\
\cline { 2 - 9 } & $\mathrm{E}$ & $\mathrm{N}$ & $\mathrm{E}$ & $\mathrm{N}$ & $\mathrm{E}$ & $\mathrm{N}$ & $\mathrm{E}$ & $\mathrm{N}$ \\
\hline $\begin{array}{c}\text { RMSE } \\
(\mathbf{m})\end{array}$ & 2,72 & 5,70 & 3,73 & 5,63 & 2,91 & 5,70 & 2,74 & 8,67 \\
\hline
\end{tabular}


Ao analisar a Tabela 5, dentre os quatro modelos, percebe-se que o Modelo de Kepler 1 apresentou resultados ligeiramente melhores em relação aos demais modelos, porém muito semelhante ao Modelo Polinomial de $1^{\circ}$ grau. Para ambos, o RMSE na componente $\mathrm{N}$ foi de pouco mais de 2 pixels, e na componente $\mathrm{E}$ de pouco mais de 1 pixel.

Para o Modelo de Kepler 2, a componente $\mathrm{N}$ apresentou-se muito semelhante ao dos Modelos de Kepler 1 e Polinomial de $1^{\text {o }}$ grau. Porém a componente E apresentou um RMSE de 1,5 pixels, sendo o pior para esta componente dentre os modelos analisados.

O resultado mais desfavorável foi obtido pelo modelo polinomial de $2^{\circ}$ grau, que embora na componente $\mathrm{E}$ tenha apresentado uma acurácia concordante com os Modelos de Kepler 1 e Polinomial de $1^{\circ}$ grau, na componente $\mathrm{N}$ apresentou um RMSE de aproximadamente 3,5 pixels.

A seguir é apresentada a Figura 8 que indica graficamente o comportamento das resultantes planimétricas dos RMSE para os modelos analisados. Na legenda do gráfico tem-se K1 como o modelo de Kepler 1, K2 como o modelo de Kepler 2, P1 como o modelo polinomial de $1^{\circ}$ grau e P2 o modelo polinomial de $2^{\circ}$ grau.

Figura 8 - RMSE nos modelos na cena da área de estudo 1.

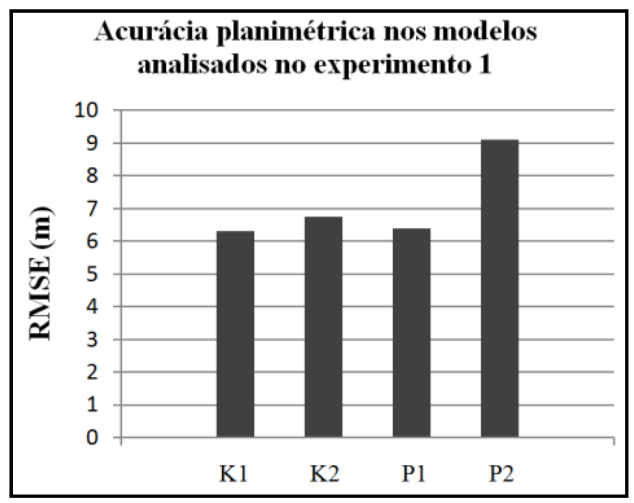

\subsection{Experimento 2}

O experimento 2 é concernente a apresentação dos resultados na área de estudo 2, parte do Distrito Federal. Este experimento caracteriza-se por uma maior quantidade de pontos de controle e verificação, distribuídos sobre a cena inteira, sem recortes. A Tabela 6 a seguir apresenta os resultados dos ajustamentos.

Para todos os modelos analisados, considerando uma precisão estimada nas observações de 3 pixels, a hipótese básica de $\hat{\sigma}_{0}^{2}=\sigma_{0}^{2}$ não é rejeitada ao nível de significância de 5\%. Os RMSE das componentes dos resíduos mostraram-se adequados à precisão adotada das observações. 
Tabela 6 - Resultados obtidos a partir dos ajustamentos na área de estudo 1.

\begin{tabular}{c|c|c|c}
\hline $\begin{array}{c}\text { Modelo } \\
\text { Polinomial de 1 } \\
\text { grau }\end{array}$ & $\begin{array}{c}\text { Modelo } \\
\text { Polinomial de 2 } \\
\text { grau }\end{array}$ & $\begin{array}{c}\text { Modelo de } \\
\text { Kepler 1 }\end{array}$ & $\begin{array}{c}\text { Modelo de } \\
\text { Kepler 2 }\end{array}$ \\
\hline$\hat{\sigma}_{0}^{2}=0,8286$ & $\hat{\sigma}_{0}^{2}=0,8368$ & $\hat{\sigma}_{0}^{2}=0,6198$ & $\hat{\sigma}_{0}^{2}=0,6228$ \\
$\chi^{2}$ calculado: & $\chi^{2}$ calculado: & $\chi^{2}$ calculado: & $\chi^{2}$ calculado: \\
109,381 & 107,111 & 80,5800 & 80,346 \\
$\chi^{2}$ teórico & $\chi^{2}$ teórico & $\chi^{2}$ teórico & $\chi^{2}$ teórico \\
$(132 ; 0,975):$ & $(128 ; 0,975):$ & $(130 ; 0,975):$ & $(129 ; 0,975):$ \\
165,696 & 161,2089 & 163,453 & 162,331 \\
\hline RMSE $V x_{s}=0,027$ & RMSE $V x_{s}=0,026$ & RMSE $V x_{s}=0,026$ & RMSE $V x_{s}=0,026$ \\
mm & $m m$ & $m m$ & mm \\
\hline RMSE $V y_{s}=0,027$ & RMSE $V y_{s}=0,026$ & RMSE $V y_{s}=0,019$ & RMSE $V y_{s}=0,019$ \\
mm & mm & mm & mm \\
\hline
\end{tabular}

De igual forma, para os três modelos ocorreu a avaliação da acurácia dos ajustamentos utilizando-se os modelos de Colinearidade inversos e os parâmetros estimados (Tabela 7).

Tabela 7 - Acurácia nas componentes E, $\mathrm{N}$ dos pontos de verificação na área de estudo 2 para os modelos utilizados.

\begin{tabular}{c|c|c|c|c|c|c|c|c}
\hline \multirow{2}{*}{ Modelos } & \multicolumn{2}{|c|}{$\begin{array}{c}\text { Modelo de } \\
\text { Kepler 1 }\end{array}$} & \multicolumn{2}{c|}{$\begin{array}{c}\text { Modelo de } \\
\text { Kepler 2 }\end{array}$} & \multicolumn{2}{c|}{$\begin{array}{c}\text { Modelo } \\
\text { Polinomial de } \\
\mathbf{1}^{\mathbf{a}} \text { ordem }\end{array}$} & \multicolumn{2}{|c}{$\begin{array}{c}\text { Modelo } \\
\text { Polinomial de 2 } \\
\text { ordem }\end{array}$} \\
\cline { 2 - 8 } & $\mathrm{E}$ & $\mathrm{N}$ & $\mathrm{E}$ & $\mathrm{N}$ & $\mathrm{E}$ & $\mathrm{N}$ & $\mathrm{E}$ & $\mathrm{N}$ \\
\hline $\begin{array}{c}\text { RMSE } \\
(\mathbf{m})\end{array}$ & 3,45 & 5,66 & 3,44 & 5,67 & 4,95 & 6,24 & 4,99 & 6,31 \\
\hline
\end{tabular}

A partir da Tabela 7 observa-se que neste experimento o Modelo de Kepler 1 e 2 apresentaram resultados semelhantes, com os RMSE na componente E e $\mathrm{N}$ de aproximadamente 1,4 pixels e 2,3 pixels respectivamente.

Os modelos polinomiais de $1^{\circ}$ e $2^{\circ}$ grau apresentaram também resultados semelhantes entre si, porém, menos acurados em relação aos Modelos de Kepler 1 e 2. Nas componentes E e N, os valores de RMSE foram de aproximadamente 1,8 e 2,5 pixels respectivamente.

A Figura 9 a seguir indica graficamente o comportamento das resultantes planimétricas dos RMSE para os modelos analisados. As legendas são as mesmas das utilizadas na Figura 8. 
Figura 9 - RMSE nos modelos na cena da área de estudo 2.

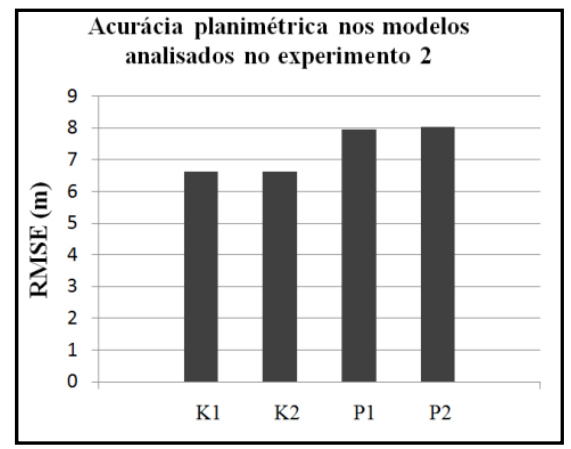

\subsection{Discussões}

Como era esperado, os valores acima de 1 pixel nas componentes do RMSE na análise dos pontos de verificação ocorreram, como nos demais trabalhos relacionados no item 2 . Isso devido em parte à baixa qualidade radiométrica das imagens, o que impõe dificuldades na identificação das feições de controle. Nesta pesquisa, os intervalos dinâmicos das imagens da área de estudo 1 e 2, com 8 bits, foram de aproximadamente 60 e 55 níveis de cinza respectivamente, conforme ilustrado na Figura 10.

Figura 10 - Histogramas das imagens da área de estudo (a) 1 e (b) 2.

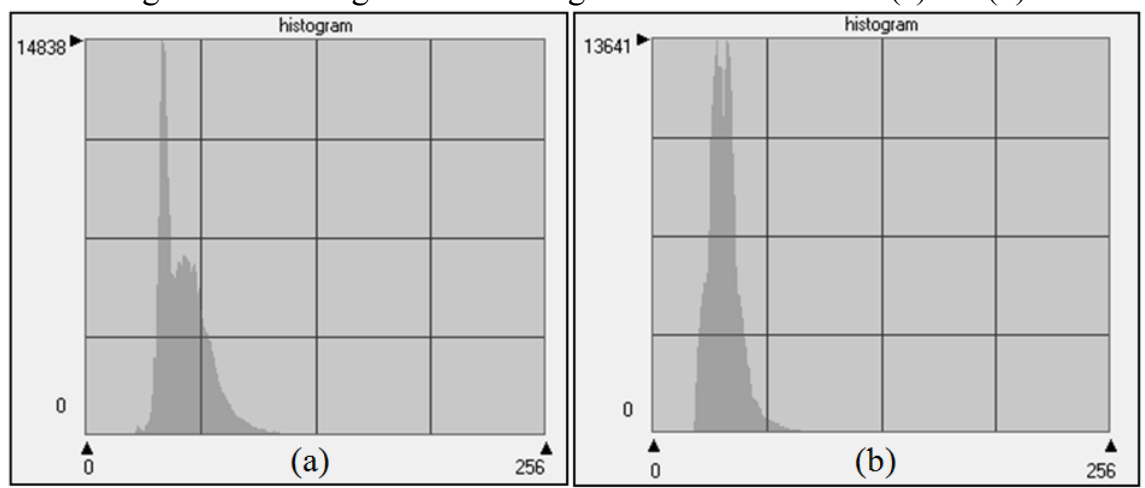

Outra questão já esperada e que ocorreu foi o maior RMSE na componente $\mathrm{N}$ dos pontos de verificação, devido ao erro sistemático não modelado do deslocamento da matriz central de detectores em relação às demais matrizes, como ilustra a Figura 2. 


\section{CONCLUSÃO}

Os resultados encontrados nesta pesquisa, de uma forma geral, concordam com os resultados obtidos em Tommaselli et al (2009), Medeiros et al (2009), Marcato et al (2010), Marcato et al (2011) e Marcato (2011), no que se refere à ordem de grandeza dos RMSE dos pontos de verificação e à identificação das questões citadas no item 2.

No contexto desta pesquisa, na análise das acurácias, o Modelo de Kepler 1, além de requisitar uma quantidade menor de pontos de controle, obteve melhores resultados, tanto para a situação de poucos pontos de controle, quanto para uma grande quantidade de pontos de controle. Para a situação de poucos pontos de controle na imagem recortada (área de estudo 1), os dois modelos de Kepler e o modelo polinomial de $1^{\circ}$ grau apresentaram resultados semelhantes.

Em relação às acurácias obtidas nos experimentos, no primeiro os resultados foram melhores. Isto pode estar associado ao fato da cena ter sido recortada, ou seja, possuir um menor número de linhas, o que representa um menor intervalo de tempo na trajetória do satélite. $\mathrm{O}$ fato de o tempo ser menor indica que a trajetória é mais próxima de uma função linear e, neste caso, o modelo linear representou mais adequadamente o trecho.

Para trabalhos futuros sugere-se a realização da fototriangulação em imagens HRC coletadas ao longo da órbita, na mesma data. Este procedimento, realizado por Michalis e Dowman (2005), visa diminuir a quantidade de parâmetros a serem estimados no ajustamento simultâneo por feixes perspectivos. Recomenda-se ainda que seja incluída na fototriangulação a modelagem do desalinhamento entre as matrizes de detectores.

\section{AGRADECIMENTOS:}

Os autores agradecem ao Curso de Pós-Graduação em Ciências Geodésicas CPGCG/UFPR, à agência de fomento CAPES, pela concessão da bolsa de estudos e incentivo à pesquisa. Ao INPE pelo fornecimento das imagens HRC - CBERS2B em nível de processamento 1.

\section{REFERÊNCIAS BIBLIOGRÁFICAS}

AL-ROUSAN, N.; CHENG P., PETRIE, G.; TOUTIN, T.; VALADAN ZOEJ, M. J. Automated DEM Extraction and Orthoimage Generation from SPOT Level 1B Imagery. Photogrammetric Engineering and Remote Sensing, v. 63, n. 8, p. 965-974, 1997.

BAIOCCHI V.; CRESPI M.; DE VENDICTIS L.; GIANNONE F. A new rigorous model for the orthorectification of synchronous and asynchronous high resolution imagery, In: Proceedings of the 24th EARSeL Symposium, 24. Dubrovnik, pp. 461-468. 2004.

ANG, K. I.; CHO, W. Analysis of EOC Sensor Model. In: FIG WORKING WEEK, 2001, Seoul, Korea. New Technology for a New Century, Seoul, p. 57-65, 2001. 
CARVAlHO, L. A. S.; STRAUSS C.; FONSECA, L. M. G. Determinação da resolução efetiva da câmera HRC-CBERS-2B pelo método de espalhamento de borda. In: Simpósio Brasileiro de Sensoriamento Remoto, XIV, 2009, Natal. Anais do XIV SBSR. São José dos Campos. INPE, p. 1975-1982.

CHEN, L.C.; TEO, T.A. Rigorous Generation of Orthophotos from EROS-A High Resolution Satellite Images. International Archives of Photogrametry and Remote Sensing and Spatial Information Sciences, v. 34, n. B4, p. 620-625, 2002.

DOWMAN, I.; MICHALIS, P.; LI, Z. Analysis of Urban Landscape Using Multi Sensor Data. Disponivel em:< http://repository.tksc.jaxa.jp/help/pdf/SP-11007E/pdf/PI061_Ian_Dowman.pdf> Acessado em: 20 de dezembro de 2011.

EPIPHANIO, J. C. N. CBERS: estado atual e futuro. In: Simpósio Brasileiro de Sensoriamento Remoto, XIV, 2009, Natal. Anais do XIV SBSR. São José dos Campos. INPE, p. 2001 - 2008.

FRITSCH, D.; STALLMANN, D. Rigorous photogrammetric modelling processing of high-resolution satellite imagery. In: ISPRS, 33. 2000, Amsterdam. International Archives of Photogrammetry and Remote Sensing, Amsterdam: ISPRS, p. 313-321, 2000.

GALO, M. Fotogrametria III - Notas de aula. Disciplina do Curso de Graduação em Engenharia Cartográfica da UNESP de Presidente Prudente, 2006.

GIANONE, F. A rigorous model for High Resolution Satellite Imagery Orientation. $\mathrm{PhD}$ thesis (PhD em Engenharia civil), Department of "Idraulica e Trasporti e Strade", "Geodesia e Geomatica" Area, University of Rome, Roma, 2006.

GUGAN, D. J.; DOWMAN, I. J. Topographic mapping from SPOT imagery. Photogrammetric Engineering \& Remote Sensing, v. 54, n. 10, p. 1409-1414, 1988.

GUPTA, R.; HARTLEY, R. I. Linear Pushbroom Cameras. IEEE Transactions on Pattern Analysis and Machine Intelligence, v. 19, n. 9, p. 963-975, 1997.

JACOBSEN, K. Orientation of high resolution optical space images. In: ASPRS 2007 Annual Conference, Tampa, 2007, Florida. Proceedings... Tampa, Flórida, 2007.

KIM, T.; DOWMAN, I. Comparison of two physical sensor models for satellite images: Position-Rotation model and Orbit-Attitude model. The Photogrammetric Record, v. 21, n. 114, p. 110-123, 2006.

KRATKY, V. Rigorous photogrammetric Proessing of SPOT Images at CCM Canada. ISPRS Journal of Photogrametry and Remote Sensing, p. 53-71, n. 44, 1989.

MARCATO, J. J. Fototriangulação em bloco de imagens orbitais com modelos rigorosos baseados em pontos e retas. Dissertação (Mestrado em Ciências Cartográficas) - FCT, Universidade Estadual de São Paulo, Presidente Prudente. 2011.

MARCATO J. J.; TOMMASELLI, A. M. G.; MEDEIROS, N. G.; OLIVEIRA, R. A. Bundle block adjustment of CBERS 2B HRC images using control lines In: 
Canadian Geomatics Conference 2010 and the International Symposium of Photogrammetry and Remote Sensing Commission I, 2010, Calgary. Proceedings. Calgary: Canadian Institute of Geomatics and ISPRS, 2010.

MARCATO, J. J.; TOMMASELLI, A. M. G.; OLIVEIRA, R. A.; MEDEIROS, N. G.; MARCATO, V. J. Orientação de imagens CBERS-2B usando o modelo rigoroso de colinearidade com dados orbitais. Boletim de Ciências Geodésicas, v.17, n. 3, p. 401-416, 2011.

MEDEIROS, N. G.; TOMMASELLI, A. M. G. Orientação indireta de imagens CBERS: Avaliação de técnicas que usam linhas retas e sua combinação com pontos. Boletim de Ciências Geodésicas, v.15, n. 1, p. 33-57, 2009.

MEDEIROS, N. G.; TOMMASELLI, A. M. G.; MARCATO JUNIOR, J. Orientação Exterior de Imagens Orbitais HRC-CBERS2B com Modelo de Coplanaridade Usando Feições Retas. In: Colóquio Brasileiro de Ciências Geodésicas, IV, 2009, Curitiba. Anais do IV do CBCG. Curitiba. UFPR, p. 1-2.

MICHALIS P.; DOWMAN I.J. A model for along track stereosensors using rigorous orbit mechanics. International Archives of the Photogrammetry, Remote Sensing and Spatial Information Sciences, v. 36, n. 1/W1, 2005.

OLIVEIRA, R. A. MARCATO, J. J.; TOMMASELLI, A. M. G. Fototriangulação de imagens CBERS-2B HRC com injunções de posição da plataforma. In: Simpósio Brasileiro de Sensoriamento Remoto, XV, 2011, Natal. Anais do XV SBSR. São José dos Campos. INPE, p. 2468 - 2475.

ORUN, A. B.; NATARAJAN, K. A Modified Bundle Adjustment Software for SPOT Imagery and Photography: Tradeoff. Photogrammetric Engineering and Remote Sensing, v. 60, n. 12, p. 1431-1437, 1994.

RADHADEVI, P. V.; RAMACHANDRAN, R.; MURALIMOHAN, A. S. R. K. V. Restitution of IRS-1C PAN data using an orbit attitude model and minimum control. Journal of Photogrammetry and Remote Sensing, v. 53, n. 5, p. 262271, 1998.

SALAMONOWICZ, P. H. Satellite orientation and position for geometric correction of scanner imagery. Photogrammetric Engineering \& Remote Sensing, v. 52, n. 4, p. 491-499, 1986.

SEEBER, G. Satellite geodesy: foundations, methods, and applications. Berlim: Walter de Gruyter, 2003.

TOMMASELli, A. M. G.; OLIVEIRA, R. A.; MARCATO JUNIOR, J.; MEDEIROS, N. G. Avaliação dos resultados da fototriangulação de imagens orbitais coletadas pelo sensor HRC do satélite CBERS-2B. In: Colóquio Brasileiro de Ciências Geodésicas, IV, 2009, Curitiba. Anais do IV do CBCG. Curitiba. UFPR, p. 1-2.

TOUTIN, T. Geometric processing of remote sensing images: models, algorithms and methods. International Journal of Remote Sensing, p. 1893-1924, 2004.

VALADAN ZOEJ, M. J.; PETRIE, G. Mathematical Modeling and Accuracy Testing of SPOT Level 1B Stereo-Pairs, Photogrammetric Record, v. 16, n. 91, p. 67-82, 1998. 
VALADAN ZOEJ, M. J.; SADEGHIAN, S. Orbital Parameter Modeling and Accuracy Testing of IKONOS Geo Image, Photogrammetric Journal of Finland, v. 18, n. 2, p. 70-80, 2003.

WESTIN, T. Precision rectification of SPOT imagery. Photogrammetric Engineering and Remote Sensing, v. 56, n. 2, p. 247-253, 1990.

(Recebido em maio de 2012. Aceito em março de 2013.) 